\title{
New Orders \\ In Search of a New Point-Block Diagram for Hong Kong
}

\section{OLIVIER OTTEVAERE}

University of Hong Kong
The project explores a series of alternative structures for housing through a design-research prototyping process. Nine proto-structures are developed through the conception and realization of columns cast in concrete. The series explores specific structural principles at 1to1 scale, which are further architecturally tested as speculative towers for urban living at 1to100 scale.

At 1 to 1 scale, an empirical approach is adopted for the making of the columns. Concrete as process rather than just concrete as material sets the main methodology for the design-research. Trial and error experiments, closely related to the properties of the material (liquid to solid formation), seek to put forward new techniques of formwork design and construction procedures that are more flexible and more sustainable.

Design analysis, informed by the work of the early 'structural rationalists' (E. Torroja, F. Candela, P.L. Nervi, H. Isler, R. Maillard, E. Dieste, et al.), considers the transformation of structural languages in an attempt to revive an architecture for vertical living (point-block).

While these structural mavericks took reinforced concrete to the limit of what the new material could do both structurally and spatially, their pioneering work responded, for the most part, to lower building scales and to singular programs (i.e. civic, cultural, religious); all but Housing. The research revives the dialogue in the context of high density Asian living. The dialogue is being pursued with new technology that breaks the homogenizing influence of concrete.
The modernist search for concrete-based new models for living, fuelled by an urgent need for cheap housing after the world war destruction, was devised in Western Europe in the form of a cast-in-place skeleton frame. This flexible column-slab system was first patented and universally distributed by the 'Hennebique' enterprise in the late 19th century and later, in 1914, generalized by Le Corbusier as the Domino protocol (domus-innovation). Its promise was social; to support individualization of living spaces by internally liberating the plan of a building from its structural imperatives ('Free plan'). A century later, this now ubiquitous structural system has largely achieved the reverse: mainly creating an order characterized by homogeneity based on the repetition of the same living units across building scales.

The design-research aims to develop new structural articulations for high-rises that are more agile in negotiating the transition from one kind of program to another within a complex. These aim to provide residents with gradients of communal spaces that reconcile (semi-) outdoor living issues in a sub-tropical climate.

One of the overarching research propositions is to reassert structural design and construction procedures as the main driver for new Housing organizations in a way that helps break from the monotony of current systems. 


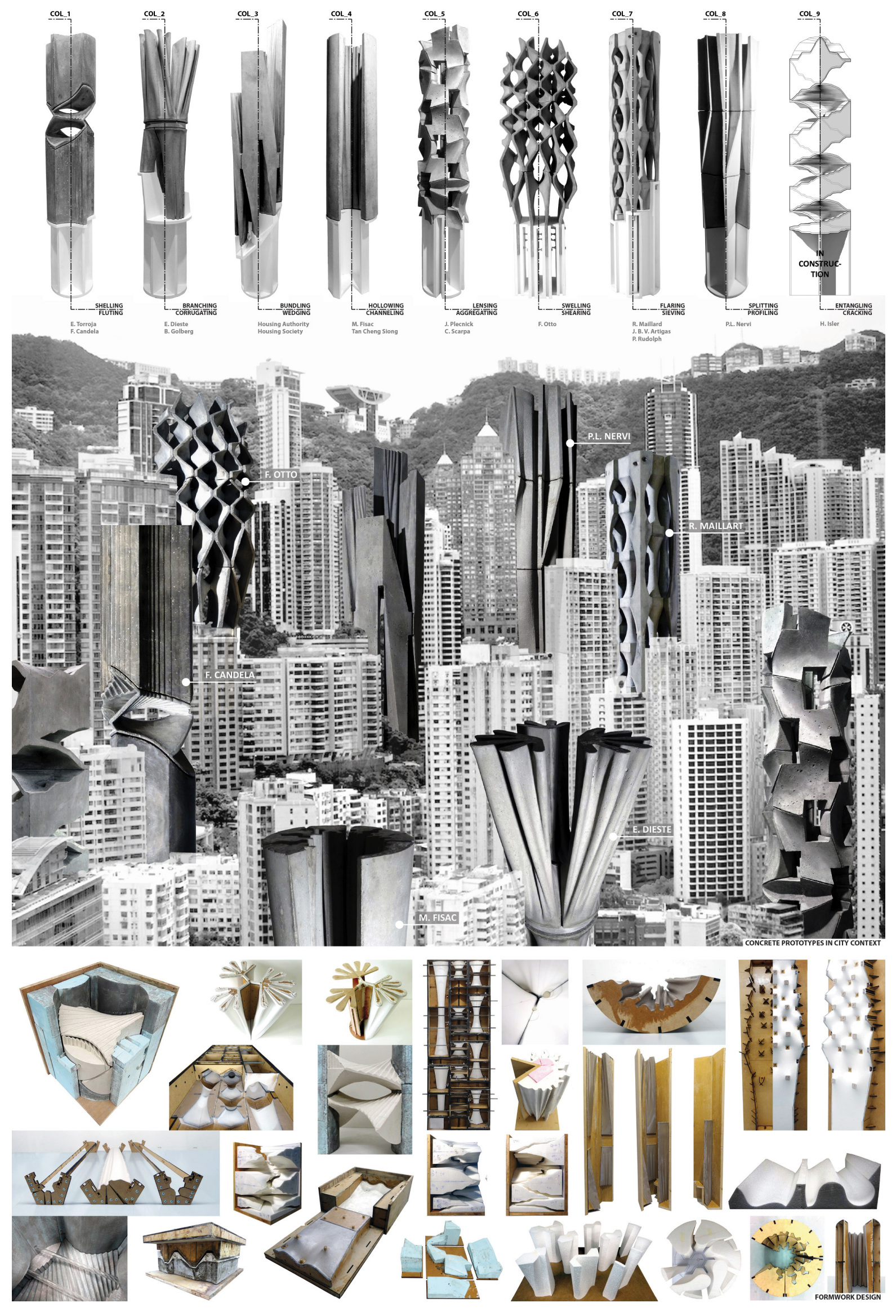

